\title{
La investigación de la comunicación en España desde la Economia Política de la Comunicación
}

The communication research in Spain from the perspective of Political Economy of Communication

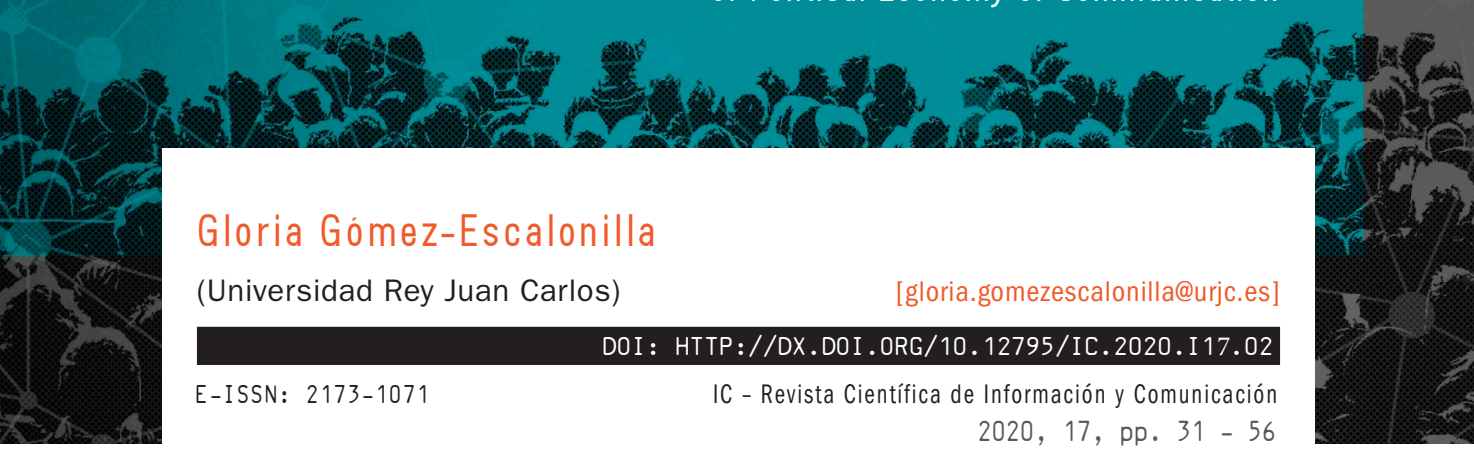

\section{Resumen}

Se presentan los resultados de un estudio que analiza tesis doctorales, proyectos financiados, comunicaciones presentadas al principal congreso científico y artículos publicados en las revistas indexadas realizados desde la perspectiva de la Economía Política de la Comunicación en los últimos diez años, concluyendo que es una línea de investigación consolidada en la investigación en comunicación que aborda especialmente la estructura y la incidencia del impacto digital en el audiovisual español.

\section{Abstract}

The results of a study that analyzes doctoral theses, funded projects, communications presented to the main scientific congress and articles published in indexed journals made from the perspective of the Political Economy of Communication in the last ten years are presented, concluding that it is a line of consolidated research in communication research that addresses especially the structure and incidence of digital impact on Spanish audiovisuals.

\section{Palabras clave}

Economía política de la comunicación; Investigación; Industrias culturales; teoría crítica; meta-investigación. 


\section{Keywords}

Political economy of communication, investigation, cultural industries, critical theory, meta-research.

\section{Sumario}

1. Introducción

2. Contexto: de la teoría crítica a la Economía Política de la Comunicación (EPC)

3. Metodología del estudio sobre la investigación desde la EPC

4. Resultados del estudio: la investigación en España desde la perspectiva de la EPC

4.1. Más autores que autoras en la práctica investigadora

4.2. Enfoques principales de la investigación desde la EPC

4.3. Medios y soportes analizados: la supremacía del audiovisual

4.4. Los diseños metodológicos de las investigaciones desde la EPC

5. Discusión y conclusiones

6. Bibliografía

\section{Summay:}

1. Introduction

2. Context: from critical theory to the Political Economy of Communication (EPC)

3. Methodology of the study on research from the EPC

4. Study results: research in Spain from the EPC perspective

4.1. More male authors than female authors in research practice

4.2. Main research approaches from the EPC

4.3. Media and supports analyzed: the supremacy of the audiovisual

4.4. The methodological designs of the investigations from the EPC

5. Discussion and conclusions

6. Bibliography 


\section{Introducción}

Este estudio forma parte del proyecto de investigación "Mapa de la Investigación en Comunicación en las Universidades Españolas de 2007 a 2018" (PGC2018-093358-B-100, Ministerio de Ciencia e Innovación) que ofrece un mapa de las prácticas científicas en comunicación realizadas en España en los últimos 10 años (www.mapcom.es). El estudio que se presenta a continuación se refiere a una dimensión muy concreta del proyecto general: la investigación que se realiza desde la Economía Política de la Comunicación (EPC).

El objetivo principal del trabajo es, pues, conocer la investigación desarrollada desde esta perspectiva crítica: lo que representa con respecto a otro tipo de enfoques y sus características principales: los investigadores, los objetos y las metodologías de investigación que se emplean en esta línea de investigación, en definitiva, quién, qué y cómo se investiga desde la EPC.

La reflexión sobre la investigación en un campo científico contribuye a su consolidación como disciplina, pero también "refleja una toma de conciencia acerca de los propios límites, métodos e incluso personas que lo integran" (Martín Algarra; Serrano y Rebolledo, 2018: 69), intentando revelar los contextos y condicionantes de esa investigación,las prácticas que generan inquietud científica y los modos en que se investiga.Por ello, el estudio de la investigación en comunicación ha sido, en sí mismo, un objeto de estudio propio de esta disciplina, que aparece en torno a los años ochenta y noventa (Moragas, 1988; Caffarel, Domínguez y Romano, 1989; De Aguilera, 1998; Jones, 1998), a lo que no fue ajeno, como señala Martínez Nicolás (2009) el "extraordinario desarrollo del sistema comunicativo español en estos años y la repercusión de la nueva estructura del Estado en el ámbito de la cultura". A medida que se van consolidando los estudios de comunicación y la comunicación como disciplina científica, esta línea de investigación incrementa las referencias (Almirón y Reig, 2007; Fernández Quijada y Masip, 2013; Díaz Nosty y De Frutos, 2016; Saperas, 2016), algunas centradas en determinadas prácticas científicas y otras en diferentes objetos o líneas de estudio, por ejemplo, la que se centra en la investigación sobre la comunicación para el desarrollo (Barranquero y Limón, 2017), aunque no abundan las que se refieren a la Economía Política de la Comunicación,un ámbito que la presente contribución intenta paliar.

La complejidad inherente a los fenómenos comunicativos ha llevado a que la investigación sobre comunicación se haya realizado desde diferentes perspectivas. 
La experiencia acumulada en la investigación de este campo permite agrupar esas miradas en tres grandes paradigmas que relacionan concepciones teóricas, supuestos ontológicos y formas particulares de acercarse a la realidad: el planteamiento positivista, el interpretativista y el crítico, siguiendo la terminología propuesta por Rodrigo Alsina (2014:83); triple clasificación que ya propusieron, aunque con diferente denominación, autores provenientes de las ciencias sociales, como Neuman (1994), Del Rincón, Arnal, Latorre y Sans (1995), y que adoptaron los primeros teóricos de la comunicación en nuestro país (Montero,1994; Saperas, 1998), gozando actualmente de cierto consenso.

Estos tres paradigmas conciben la comunicación de distinta manera. Para la investigación positivista, los fenómenos comunicativos tienen "existencia propia, independiente de quien los estudia, y están regidos por leyes, las cuales permiten explicar, predecir y controlar estos fenómenos. En consecuencia, la finalidad de las ciencias está dirigida a descubrir esas leyes" (González Morales, 2003). La investigación positivista fue, y aún sigue siendo ahora, el paradigma dominante, imperante en la Mass Communication Research, centrada en el estudio de los efectos de los medios, con una fuerte implantación en la investigación aplicada y comercial.

Por su parte, el paradigma interpretativista asume que la realidad depende de cada individuo, es una construcción social, por lo que la ciencia "busca descubrir los significados de las acciones sociales para llegar a entender e interpretar cómo la gente crea y mantiene sus mundos sociales" (Neuman, 1994: 62). Frente al empirismo de los positivistas, esta corriente propone la heurística como método del conocer y, frente alos efectos como ámbito privilegiado de estudio, se centran en la interpretación de los sujetos.

Por lo que respecta al paradigma crítico, también surgió en contraposición a los planteamientos positivistas, como señala Carrasco (2016:2): “la teoría crítica constituye uno de los marcos teóricos originarios de los estudios científicos de comunicación, en su disputa con la mass communication research (y supone) la reivindicación de una investigación de la comunicación alternativa a la tradición funcionalista dominante". Pero sus planteamientos se alejan también de los presupuestos interpretativistas, pues defienden que la realidad existe más allá de la concepción que se tiene de ella. Fieles al materialismo histórico propuesto por Marx, parten de la realidad, pero no de una realidad ajena a la sociedad, como planteaban los empiristas, sino que la sociedad forma parte de ella, sometida a las leyes de la naturaleza y de la historia, las bases 
de la dialéctica. Aplicado a los estudios de comunicación, se centran en investigar la naturaleza, configuración y desarrollo histórico y social de las formas y sistemas de comunicación de las sociedades contemporáneas.

\section{Contexto: de la teoría crítica a la Economía Política de la Comunicación}

A principios de la década de los veinte, Felix Weil crea en Frankfurt un instituto de investigación cuyo propósito es analizar la sociedad desde planteamientos marxistas. Aunque en un principio el propósito fuera analizar por qué fracasó en Alemania la revolución que triunfó en Rusia, la irrupción del nazismo centró sus esfuerzos intelectuales en explicar el ascenso de Hitler, aunque tuvieran que hacerlo en el exilio. En Estados Unidos, los investigadores de la escuela de Frankfurt continuaron su labor de estudiar las sociedades en lo que se denominó la escuela crítica, "una etiqueta para camuflar la teoría marxista" y no exponerse como pensadores comunistas(Fuchs, 2017).

Son muchos los autores y propuestas teóricas que tienen la marca del instituto, pero la aportación más importante a los estudios de comunicación es el concepto de industria cultural. En 1944, en EEUU, se publica Dialéctica de la Ilustración, donde Theodor Adorno y Max Horkheimer desarrollan este concepto en el capítulo cuatro para referirse a los nuevos medios de comunicación de masas, haciendo hincapié no solo en la reproductibilidad técnica del original que ya destacó Benjamin, también y sobre todo en su mercantilización y en el entramado industrial que genera, que funciona con el motor de su consumo: "la industria cultural puede vanagloriarse de haber llevado a cabo con energía y de haber erigido en principio la, a menudo, torpe transposición del arte en la esfera del consumo" (Horkheimer y Adorno, 1994:179). Pero este consumo, más que liberador para los públicos,implica la degradación del contenido y su capacidad alienante: “la abolición del privilegio cultural por liquidación no introduce a las masas en ámbitos que les estaban vedados; más bien contribuye, en las actuales condiciones sociales, justamente al desmoronamiento de la cultura", pues "en la industria cultural desaparece tanto la crítica como el respeto: a la crítica le sucede el juicio pericial mecánico, y al respeto, el culto efímero de la celebridad" (Horkheimer y Adorno, 1994:205). A la postre, la cultura se transforma en ideología destinada a conservar el sistema y el público deviene en un ser de consumo pasivo, fácilmente manipulable. No en vano las industrias 
culturales son, para estos autores, "la ilustración como engaño de masas" (Horkheimer y Adorno, 1994:165), tal como se titula ese capítulo que hizo historia.

A pesar de que han pasado más de setenta años desde su publicación, estos planteamientos siguen vigentes, pues la actual también es "una industria que nos ofrece más de lo mismo, desarrolla algoritmos para continuar encadenándonos a nuestros gustos, y nos hace desear nuestra propia dominación (Jeffries, 2018: 447). Por ello, en la investigación actual en comunicación, su legado continúa en una corriente teórica, la Economía Política de la Comunicación, donde los investigadores se valen del sustrato teórico propuesto por los autores de la Teoría Crítica, especialmente del potencial heurístico del concepto de industria cultural, para describir y analizar los sistemas mediáticos y las estructuras de la comunicación de las sociedades contemporáneas. Así lo destaca Bustamante (2002: 24), para quien actualmente el término de industria cultural resulta válido para expresar "la determinación mercantil e industrial de la mayor parte de la cultura contemporánea y, correlativamente, de las numerosas especificidades que hacen de estos sectores unas industrias diferentes a las restantes".

Diferentes porque su carácter cultural exige cierta regulación que garantice el acceso a la producción, especialmente de las minorías, al tiempo que garantice la mayor difusión social de la cultura; y estos principios básicos chocan con la lógica económica que prima en las sociedades actuales. Además, su dinámica industrial ha ayudado a que se genere una configuración concentrada, oligopolística o monopolizada en la mayoría de los mercados de la comunicación que justifica el sentido crítico que proponían Adorno y Horkheimer, pues desde la EPC se "denuncia la capacidad de las empresas más influyentes para manipular la opinión pública" (Artero, 2015), línea que expresó en los ochenta el informe McBride "Voces múltiples, un solo mundo", y que sigue vigente por la influencia de los estudios culturales británicos, especialmente en Latinoamérica (Medina, 1998).

Pero esta configuración del mercado también ha hecho que se ponga "el énfasis en las estructuras de los medios y el contenido, comprensible en vista de la importancia de las compañías globales de medios y el crecimiento del valor del contenido mediático" (Mosco, 2006:67). Además, los nuevos planteamientos de esta corriente no solo abren las líneas de estudio a los valores y contenidos que se vehiculan, también lo vinculan "al estudio de los consumos y al debate en torno a las políticas de medios y la organización democrática de la información y la cultura" (Sierra, 2009), con fuertes vínculos con estudios que analizan fenómenos de estructuración, como señala Mosco (2006:72) “de 
género o raza y los definidos ampliamente como movimientos sociales que, junto con la clase, constituyen muchas de las relaciones sociales de comunicación".

\section{Metodología del estudio sobre la investigación desde la EPC}

Este trabajo sigue la metodología empleada en el proyecto del que forma parte que elabora un riguroso censo de:1. proyectos de investigación (a partir de la información proporcionada por el Ministerio de Ciencia e Innovación y las propias Universidades), 2. tesis doctorales (a partir de la base de datos Teseo), 3. artículos de investigación de las primeras revistas españolas según el índice h, elaborado por Google Scholar: Comunicar, Revista Latina, Comunicación y Sociedad, Estudios del Mensaje periodístico, Telos, Zer, Trípodos, El Profesional de la información e Historia y Comunicación Socialy 4. comunicaciones presentadas y publicadas en las Actas de los congresos de la Asociación Española de Investigadores en Comunicación (AEIC): Santiago de Compostela 2008; Málaga 2010 , Tarragona 2012, Bilbao 2014, Madrid 2016 y Salamanca 2018.

El mapeo de todas estas prácticas científicas ha supuesto el registro de un total de 4.290 contribuciones, de las que se han seleccionado las que adoptan una perspectiva clara desde la Economía Política de la Comunicación, en total 320 aportaciones que constituyen el corpus de esta investigación.

De cada una de estas investigaciones se ha consignado en una ficha de análisis tres tipos de variables: las que hacen referencia a la identificación de la contribución: tipo de investigación, año de publicación, universidad, autonomía y género del investigador principal de los proyectos, primer autor de los artículos 0 comunicaciones o del doctorando en el caso de las tesis doctorales. En segundo lugar, variables referidas al objeto: tipo de comunicación analizada y tema, medio analizado y ámbito geográfico al que se acota la investigación, "que puede ser clasificado según se centre en los diferentes "espacios" comunicativos, es decir el transnacional, el estatal, el regional y el local" (Jones,1998). El tercer tipo de variables hace referencia a la metodología de investigación, consignando los métodos utilizados, clasificándolos en función de la fuente principal de datos: conversacionales, observacionales, experimentales o documentales, y anotando las técnicas de investigación utilizadas para la obtención de los datos. 


\section{Resultados del estudio: la investigación en España desde la perspectiva de la EPC}

Lo que este estudio destaca como resultado principal es que en España se investiga la comunicación desde la Economía Política, resultando una perspectiva actual dentro de las utilizadas para investigar la comunicación, aunque no es un enfoque principal. Convive con otras perspectivas, como el estudio de las representaciones 0 contenidos, el uso y consumo mediático, los efectos de la comunicación, o el estudio de los medios desde un punto de vista tecnológico, organizativo o histórico. El enfoque propio de la EPC que, como resume Almirón (2009: 564), "combina la perspectiva histórica, las relaciones de poder (o economía), el análisis estructural y una componente ética" es más bien minoritario, pues de las 4.290 contribuciones científicas sobre la comunicación que se han registrado en los últimos diez años, solo se ha adoptado este enfoque en 320 aportaciones, lo que supone el 7,5\% de la investigación en comunicación en nuestro país.

Si esta medida representa la proporción de la investigación de EPC en el conjunto de toda la investigación que se realiza en España, hay diferencias según el tipo de contribución, tal como refleja la Tabla 1. De este modo se puede decir que las tesis doctorales no son las que más suelen adoptar esta perspectiva, pues hay una diferencia de más de un punto con respecto a la media. Sin embargo, la gran cantidad de tesis que se han defendido en este periodo hace que representen más de la mitad de las contribuciones desde la EPC. El tratarse de una investigación inicial podría explicar esta pauta, pues la investigación desde esta perspectiva de la economía política requiere un bagaje teórico del que los doctorandos carecen. De hecho, se adopta con una media que se incrementa en más de tres puntos cuando se trata de la investigación de mayor nivel: los proyectos competitivos, un indicador de cierto prestigio científico.

También se muestra esa tendencia de una mayor proporción de contribuciones desde esta perspectiva en las comunicaciones a los congresos de la AEIC, aunque ello responde más bien a la existencia de una sección específica "Estructura y políticas de la comunicación", que fomenta la concurrencia de investigaciones y propuestas realizadas desde esta línea. Con respecto a la publicación de artículos publicados en revistas indexadas, no acumulan demasiadas aportaciones, el $10 \%$ del total de las investigaciones de EPC y la media de lo que representa esta perspectiva en el conjunto de la investigación, pero sí es de reseñar que las revistas donde mayor cabida tienen son las que tienen mayor 
índice de impacto, pues El profesional de la Información (Q1) y Comunicación y Sociedad (Q2) son las que publican más artículos de EPC.

TABLA 1

Tipo de contribución científica

\begin{tabular}{|c|c|c|c|}
\hline Tipo & $N^{0}$ & \% Del total de aportaciones desde la EPC & $\%$ del tipo de contribución \\
\hline Articulos & 30 & $9,4 \%$ & $7.3 \%$ \\
\hline Comunicaciones & 86 & $26,9 \%$ & $10,11 \%$ \\
\hline Proyectos $1+D+1$ & 26 & $8,1 \%$ & $10,4 \%$ \\
\hline Tesis Doctorales & 178 & $55,6 \%$ & $6.3 \%$ \\
\hline
\end{tabular}

\subsection{Más autores que autoras en la práctica investigadora}

Lo primero que hay que señalar cuando se quiere caracterizar a los autores de la investigación desde esta perspectiva es que son, fundamentalmente, investigadores. A pesar de que en el conjunto de la investigación en comunicación en España se ha logrado el equilibrio de género (Lozano, Gaitán, Caffarel y Piñuel, 2020:9), en la investigación desde la EPC priman los autores sobre las autoras, pues de las 320 aportaciones científicas analizadas, ellos firman 172, prácticamente el $56 \%$, y ellas 139 , el $44 \%$, lo que supone que la brecha de género es de más de 10 puntos porcentuales.

Esa diferencia varía en función del tipo de contribución que sea, pues si en todas se reproduce la mayor presencia de varones sobre mujeres, hay una mayor diferencia en la firma de los artículos indexados, donde la diferencia supera los veinte puntos y, sobre todo, en los proyectos I+D+i, que supera con creces los treinta puntos de diferencia, por lo que no solo hay brecha de género, también existe techo de cristal, menor presencia de mujeres según se avanza en la jerarquía científica. 
TABLA 2

Relación de investigadores e investigadoras según las contribuciones

\begin{tabular}{lc|c|c|c|c}
\hline & Tesis & Comunicaciones & Articulos & Proyectos & Todas \\
\hline Mujeres & $48,3 \%$ & $43,0 \%$ & $38,1 \%$ & $30,8 \%$ & $44 \%$ \\
\hline Varones & $51,7 \%$ & $57,0 \%$ & $61,9 \%$ & $69,2 \%$ & $55,3 \%$ \\
\hline
\end{tabular}

También se ha analizado la universidad de procedencia del IP en el caso de proyectos competitivos, del primer autor de las comunicaciones o del centro de lectura en el caso de tesis doctorales, y lo primero que se puede decir es que se trata, en más de un $99,5 \%$ de los casos, de una universidad pública, perteneciendo uno de cada cuatro a la Universidad Complutense de Madrid, que concentra 71 aportaciones, la que más. Con mucha diferencia se encuentran otras universidades que suman más de 10 contribuciones, y son, por este orden: la Universidad Autónoma de Barcelona (23), la Universidad de Málaga (22), la Universidad Carlos III (16), la Universidad de Navarra (15) y la Universidad de Santiago de Compostela, la de Sevilla, la del País Vasco o la Rey Juan Carlos con 14 aportaciones cada una. El contar con más o menos profesores que investigan desde esta perspectiva,y más o menos prolíficos en su actividad investigadora, puede explicar esta mayor presencia de unos centros sobre otros, en todo caso todos ellos son centros de referencia, por su tradición, su dimensión o su prestigio investigador, lo que indica cierto prestigio científico de la investigación realizada desde esta perspectiva.

\subsection{Enfoques principales de la investigación desde la EPC}

El principal enfoque que prima en la investigación desde la Economía Política de la Comunicación es el análisis de la estructura mediática, ya sea el sistema de medios de comunicación en su conjunto o en configuraciones sectoriales determinadas, por ejemplo, el duopolio televisivo español, la televisión de pago, los canales temáticos económicos, medios alternativos, la prensa gratuita, la prensa gráfica o los sistemas de medios locales, así ha sido en 86 estudios, lo que supone el $27 \%$ de todas las contribuciones desde esta perspectiva. 
El otro enfoque prioritario es el que analiza la incidencia en las industrias de la comunicación de las nuevas tecnologías, aspecto sobre el que se han centrado el $18,5 \%$ de las investigaciones. En un escenario como el comunicativo, donde la digitalización ha supuesto toda una revolución, es hasta cierto punto esperable que la investigación visualizara este cambio. La preeminencia del enfoque tecnológico también ha sido destacado por otros autores, cuando dicen que "la investigación crítica quedaría, por tanto, sometida a los márgenes de la cuádruple raíz ya definida del nuevo arquetipo cultural: cultura-tecnología-comunicación-mercado" (Carrasco y Saperas, 2013).

Cierto protagonismo ha tenido también la investigación que analiza algún aspecto del mercado (16,3\%). En 52 investigaciones se ha estudiado la distribución, exhibición, los modelos de negocio, estrategias de crecimiento u otros aspectos de la comercialización de la comunicación.

En esta perspectiva suele ser habitual realizar un acercamiento a un caso particular, cuando se analiza en profundidad un medio de comunicación, modalidad que ha concentrado el $13,5 \%$ de las investigaciones. De este modo se han abordado estudios sobre Vocento, Prisa, Canal Parlamento, Veo Televisión, Netflix, Antena 3 Radio, Kiss FM, 20 minutos, La sexta, RTVE o los diferentes canales autonómicos, como Canal Sur, ETB o el canal autonómico valenciano Canal Nou, cuyo cierre y nueva apertura generaron cierta inquietud científica.

Otros enfoques especialmente relevantes dentro de las investigaciones de EPC son los que analizan las políticas públicas de comunicación (38 investigaciones),al fin y al cabo, la Economía Política es "una disciplina que conecta las microestructuras empresariales y los productos periodísticos con la historia y la evolución de la sociedad y sus relaciones de poder" (Galletero, 2018: 7).

También se destaca como enfoque el estudio delos medios como industrias culturales (32 aportaciones), cuando se destacan esos aspectos de comercialización e industrialización en medios determinados (PRISA, ETB...) o en un tipo de industrias, como en la industria audiovisual o en la de los videojuegos. Este enfoque se distingue del que destaca los aspectos empresariales de los medios (10 casos)dado que en esta perspectiva“cabría distinguir, por un lado, las que responden a una perspectiva crítica y, por otro lado, las que responden más a lo que podríamos calificar como lógica empresarial" (De Aguilera, 1998: 9). Finalmente, también se han destacado de manera diferencial las 10 investigaciones que han abordado específicamente las consecuencias 
de la crisis económica, por el gran impacto que han tenido en las empresas de comunicación, especialmente en la prensa.

\section{FIGURA 1}

Principales enfoques de las investigaciones desde la EPC

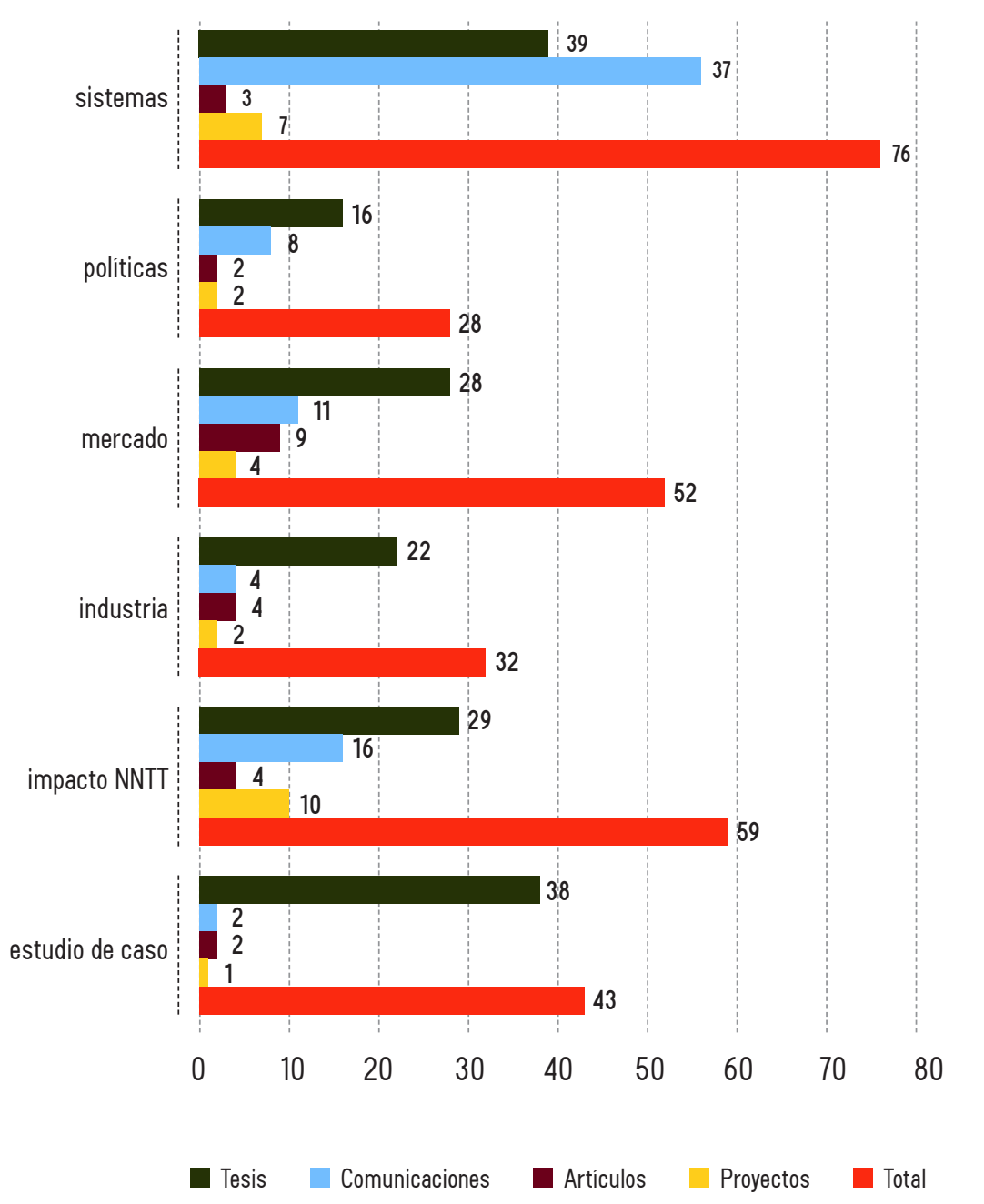


En el cruce de los enfoques principales que caracterizan las investigaciones desde esta perspectiva con el tipo de contribución, se detectan ciertas pautas que resultan significativas. Así por ejemplo cabe destacar que los estudios de caso son más característicos de las Tesis Doctorales que de otro tipo de investigaciones. Suele ser habitual, dada la limitación material para extender la investigación,que las investigaciones doctorales se centren en objetos de estudio concretos y acotados, como puede ser el estudio de un medio de comunicación que se investiga en profundidad utilizando el enfoque de caso, o bien se analicen aspectos de su configuración como industria cultural, enfoque en el que también destacan las tesis doctorales.

Otros enfoques más ambiciosos, como el análisis de sistemas comunicativos o los aspectos de mercado, o enfoques más complejos, como la incidencia en el sector comunicativo de las nuevas tecnologías, suelen ser minoritarios en las investigaciones doctorales, enfoques que suelen ser precisamente más característicos de otros tipos de investigación de mayor nivel, como los proyectos competitivos y los artículos indexados. Con respecto a las comunicaciones, se suelen centrar en acercamientos que analizan el sistema de medios o el impacto de las nuevas tecnologías de la información, las redes 0 la digitalización en los fenómenos comunicativos.

Para enmarcar el objeto de estudio, también resulta pertinente preguntarse por el ámbito de referencia geográfico al que aluden, lo que Mosco llama "espacialización" (2006:69). Pues bien, como se puede ver en la Figura 3, casi tres cuartas partes de los trabajos tienen como ámbito de referencia el marco nacional, en este caso, España, en concreto 187 aportaciones. Ello es hasta cierto punto lógico dado que es el ámbito natural de los estudios económicos, ya que así funcionan los mercados, al menos hasta ahora, pues los procesos de globalización, digitalización e internacionalización hacen que el marco convencional del estado-nación haya sido superado por las categorías supranacionales. De hecho, un $21 \%$ de los estudios se refieren a una esfera internacional, bien porque se refieran a esos espacios supranacionales, como la Unión Europea, el espacio Mediterráneo, relaciones con América Latina o las relaciones globales y mundiales, o bien porque los estudios se refieran a otros países, destacando los países latinoamericanos. Ello responde a la cercanía idiomática y cultural con los países del otro lado del Atlántico cuyos investigadores suelen recalar en España para doctorarse, y eligen sus países de origen como ámbito geográfico de su investigación doctoral, o para compartir sus investigaciones en los diferentes congresos de la AE-IC, porque el espacio internacional destaca en las tesis doctorales y en las comunicaciones mientras que los 
proyectos competitivos y los artículos de investigación se refieren, la mayoría, al ámbito nacional español.

\section{FIGURA 3}

\section{Ámbito geográfico al que se refieren los estudios desde la EPC}

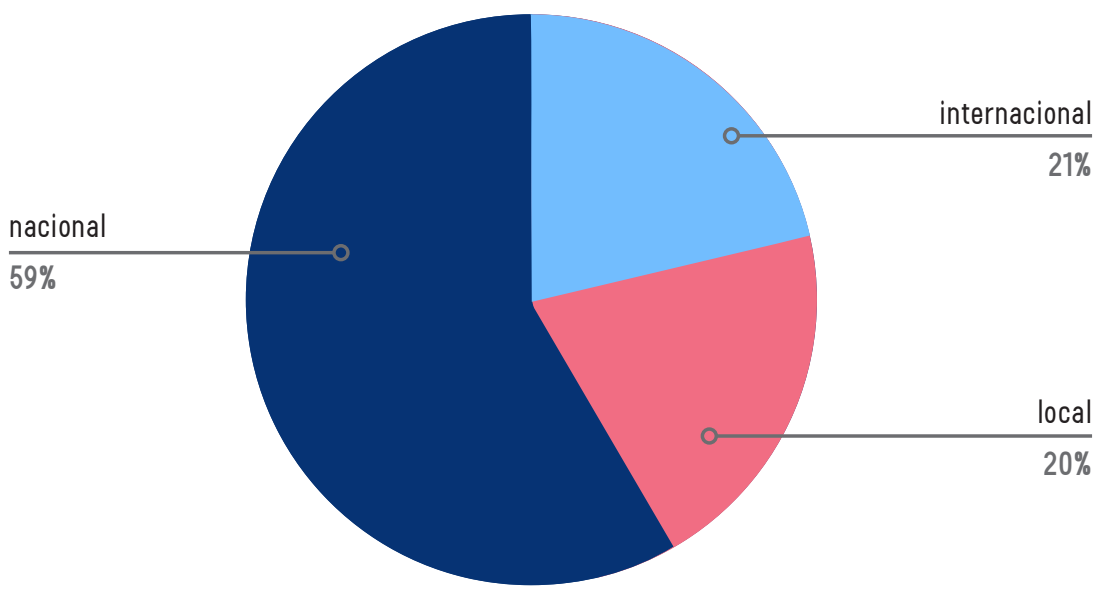

A los estudios nacionales e internacionales hay que añadir otros 64 estudios que se refieren a un ámbito más regional o local, normalmente centrados en aproximaciones que acotan comunidades autónomas de la geografía española, aunque también hay alguna aportación que se refiere a ámbitos geográficos más reducidos, como regiones o ciudades. La mayoría de estas aportaciones son tesis doctorales o comunicaciones presentadas a los diferentes congresos de la AEIC.

\subsection{Medios y soportes analizados: la supremacía del audiovisual}

Además del enfoque que se estudia, también se pueden diferenciar estos estudios en función del medio o soporte que se investiga. Hay aportaciones, en concreto 43 investigaciones, que se refieren a la comunicación como expresión genérica, que 
no concretan o se refieren a ningún medio en particular, sino que aplican el estudio al genérico mediático. Suelen ser, en su mayoría, las aportaciones de las comunicaciones a los congresos de la AEIC. Estas investigaciones representan el 13,5\% del total de las aportaciones realizadas desde esta perspectiva.

El resto sí refieren el estudio de medios o soportes más específicos, y en este tipo de acercamientos priman los medios audiovisuales. La preeminencia de lo audiovisual que han favorecido los fenómenos de convergencia mediática en el escenario comunicativo tiene su correspondencia en el predominio del audiovisual en la investigación en comunicación, pues cerca del $60 \%$ de las investigaciones realizadas desde la perspectiva de la EPC se centran en los medios audiovisuales. Ahora bien, dentro de estos medios se pueden distinguir diferentes categorías, hay investigaciones que se refieren al audiovisual genérico ( $7,5 \%$ de los casos) y otras investigaciones que ya especifican medios concretos, dentro de los cuales destaca como referencia principal el medio televisivo. Más de una de cada cuatro investigaciones se han referido a la televisión ( $27 \%$ del total): 6 proyectos competitivos, 8 artículos en revistas indexadas, 20 comunicaciones en el congreso más importante del área y 52 tesis doctorales dan cuenta de su importancia. Los cambios acaecidos en el panorama televisivo, con la concentración oligopolística en España, la irrupción de la TDT o los cambios de estructura en la televisión local han sido hitos que han provocado numerosas investigaciones de todos los tipos, desde comunicaciones y tesis doctorales a proyectos competitivos y artículos de investigación.

Le sigue en importancia el cine. La industria cinematográfica ha acaparado el $14,5 \%$ de las contribuciones desde esta perspectiva, en concreto 46 aportaciones: 2 proyectos competitivos, 5 artículos de investigación, 6 comunicaciones a los congresos de Ia AEIC, relativamente pocas, y 33 tesis doctorales sobre este medio que sigue cautivando el interés investigador del área.

La radio también se mantiene en la agenda investigadora, con el $8 \%$ de las investigaciones desde esta perspectiva, siendo en su inmensa mayoría tesis doctorales (14) y comunicaciones (10) que, sumado al único artículo publicado sobre la radio, completan las investigaciones realizadas sobre este medio.

La irrupción de las plataformas digitales en el mercado audiovisual español, cuyo paradigma es Netflix, también se ha dejado notar en el panorama investigativo, pues estos nuevos fenómenos audiovisuales han tenido una presencia notable en la investigación desde esta perspectiva, y no tanto por su peso cuantitativo, 9 investigaciones en total, sino por haber sido el tema de 3 proyectos competitivos de investigación. 
TABLA 3

Soporte de comunicación en el que se centran las investigaciones de EPC

\begin{tabular}{|c|c|c|}
\hline Tipo de medio analizado & $\mathrm{N}^{0}$ & $\%$ \\
\hline Medios (en general) & 43 & $13,5 \%$ \\
\hline Audiovisuales & 190 & $59,3 \%$ \\
\hline Audiovisual general & 24 & $7,5 \%$ \\
\hline Cine & 46 & $14,5 \%$ \\
\hline TV & 86 & $27 \%$ \\
\hline Radio & 25 & $8 \%$ \\
\hline Ficción (plataformas) & 9 & $3 \%$ \\
\hline Prensa & 62 & $19,5 \%$ \\
\hline Redes sociales & 6 & $2 \%$ \\
\hline Otros (publicidad, música, libro, videojuegos...) & 14 & $5,7 \%$ \\
\hline
\end{tabular}

A pesar de que el audiovisual ha concentrado mayor número de investigaciones realizadas desde esta perspectiva, la prensa también ha supuesto cerca del $20 \%$ del esfuerzo investigador: 5 proyectos competitivos, 11 artículos de investigación, además de 17 comunicaciones y 29 tesis doctorales da cuenta de esta importancia. No todas estas aportaciones se refieren a los medios impresos, la categoría se ha ampliado a los nuevos cibermedios, los medios periodísticos que han dado el salto al mundo digital.

De hecho, se ha valorado de manera diferenciada si el escenario investigado se refería al mundo off line $u$ on line, y aunque el predominio del ámbito convencional es mayoritario, pues refiere de manera exclusiva 158 de las 390 investigaciones registradas desde la EPC, el mundo digital también tiene una notable presencia, acaparando 71 investigaciones que se refieren a medios exclusivamente digitales o 56 aportaciones que se refieren a ambos escenarios. Hay que tener en cuenta que la importancia de los medios digitales que se observa actualmente no sucedía en los primeros años que abarca el estudio, y hasta cierto punto se puede comprobar pues desde 2015 se concentran la mitad de las investigaciones que se centran en medios digitales. $Y$ de los medios digitales 
destacan las redes sociales, se puede decir que en estos últimos diez años se ha producido una nueva revolución digital en la comunicación que corresponde a la irrupción de las redes sociales, pues estos nuevos medios han sido el objeto de 6 aportaciones, todas ellas en los últimos años, una de ellas un proyecto I+D+i.

FIGURA 2

\section{Objetos on line y off line en los acercamientos desde la EPC}

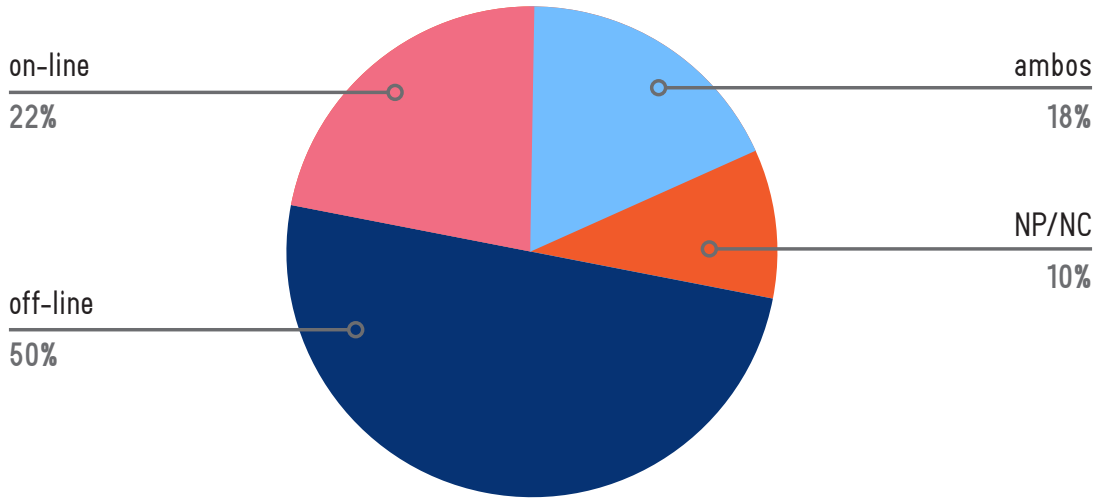

\subsection{Los diseños metodológicos de las investigaciones desde la EPC}

Sobre la metodología de investigación propia de estos estudios hay que partir de los planteamientos de los teóricos de la escuela de Frankfurt que criticaron la lógica científica que seguían los empiristas, aunque no se dedicaron a consensuar un procedimiento alternativo de producir conocimiento científico. Esta falta de explicitación por las cuestiones más procedimentales del método ha sido el blanco de la mayoría de sus críticas, baste como ejemplo la crítica que hizo Popper del método de la teoría crítica, llamado por él "historicismo", por su pretendida proyección práctica, por su comprensión intuitiva, por la pretensión holista, por su negativa al método experimental y por utilizar "un método fragmentario sin su carácter cauto y autocrítico"(Popper, 2006: 92).

Más allá de la disputa filosófica, que evidencia la distinción entre los diferentes paradigmas, es posible identificar ciertas características del modo de hacer investigación 
desde este tipo de estudios, y una primera nota diferencial es que se caracterizan por cierta indefinición metodológica, tal como señala la Tabla 4: en un 13\% de las investigaciones no se define la estrategia de investigación.

Es también minoritaria la utilización de metodologías convencionales: anecdótica la aplicación de métodos experimentales, solo en dos ocasiones, y también mínima la utilización de métodos observacionales, solo el 9\% de las 390 investigaciones analizadas, siendo la mayoría con observación sistemática, que, sumado al 7\% de los casos en los que se utilizan las encuestas, dan una dimensión relativamente escasa del peso de las técnicas de investigación cuantitativa más utilizadas en la investigación social.

TABLA 4

Métodos y técnicas de la investigación desde la EPC

\begin{tabular}{|c|c|c|c|}
\hline & & $\mathrm{N}^{0}(320)$ & $\%(100)$ \\
\hline Métodos & Conversacionales & 50 & $15,6 \%$ \\
\hline \multirow[t]{3}{*}{ Técnicas } & Encuestas & 22 & $6,8 \%$ \\
\hline & Entrevistas & 30 & $7,6 \%$ \\
\hline & Delphi & 3 & $0,7 \%$ \\
\hline Métodos & Observacionales & 36 & $9,2 \%$ \\
\hline \multirow[t]{3}{*}{ Técnicas } & Observación sistemática & 31 & $7,9 \%$ \\
\hline & Observacion participante & 2 & $0,5 \%$ \\
\hline & Autoobservación & 3 & $0,7 \%$ \\
\hline Métodos & Documentales & 166 & $42,5 \%$ \\
\hline \multirow[t]{3}{*}{ Técnicas } & Análisis de Contenido & 38 & $9,7 \%$ \\
\hline & Análisis del Discurso & 28 & $7,1 \%$ \\
\hline & Documentación & 120 & $33,3 \%$ \\
\hline Métodos & Experimentales & 2 & $0,2 \%$ \\
\hline Métodos & No consta & 50 & $12,8 \%$ \\
\hline
\end{tabular}


Lo que se suele utilizar en los diseños metodológicos de las investigaciones desde la EPC son, sobre todo, los métodos documentales, en casi la mitad de las investigaciones, concretamente en el $42 \%$ de los casos. Estos métodos que obtienen los datos primarios de los documentos son los que más se utilizan en las ciencias de la comunicación, al fin y al cabo, los fenómenos comunicativos se registran y forman parte del universo documental: periódicos, revistas, programas audiovisuales, producción fílmica, archivos web, entradas de redes sociales... de este modo el análisis de contenido y, en menor medida, el análisis del discurso son las técnicas propias de las investigaciones en el campo de la comunicación. Sin embargo, no sucede esto cuando estas investigaciones se realizan desde la perspectiva de la Economía Política de la Comunicación, pues la estrategia metodológica se basa no tanto en el estudio de los documentos comunicativos sino de las fuentes documentales sobre comunicación. Apenas un $10 \%$ de las investigaciones aplica la técnica más utilizada en este campo, el análisis de contenido. En un 7\% se utilizan técnicas de análisis del discurso, la estrategia cualitativa para acercarse a los mensajes, aun cuando existe una modalidad propuesta por Van Dijk (2002) de análisis crítico del discurso que aboga por esta técnica cuando confluyen los estudios críticos con los estudios culturales para analizar precisamente la ideología que subyace en los mensajes. En todo caso, estas técnicas son minoritarias cuando se analiza, como hacen estos estudios, la comunicación como negocio, pues la estrategia de investigación que se suele seguir, en un 33\% de los casos, se basa en la documentación, en la utilización de datos secundarios como fuente principal de datos.

En realidad,la documentación y consulta de fuentes secundarias no es privativa de esta clase de estudios, pues es una de las fases del proceso de investigación científica, pero los estudios que se realizan desde esta perspectiva se sirven delos datos secundarios no solo para conformar el estado de la cuestión o los marcos contextuales básicos,también para el trabajo de campo empírico. Y es que la documentación en estos estudios no consiste solo en la revisión de la literatura científica,también analiza estudios sectoriales y económicos, normas legales y judiciales, memorias e informes empresariales e institucionales, bases de datos y estadísticas, en definitiva, variedad y disparidad de fuentes para conocer datos de autores, de catálogos, de mercados y audiencias y, sobre todo,de los medios y su filiación empresarial, una información no siempre transparente y disponible para los investigadores pero clave para conocer la estructura de la comunicación.

Esta heterogeneidad en la naturaleza de las fuentes de información, junto con la interdisciplinariedad desde la que se aborda el objeto de estudio, la variabilidad de la 
naturaleza y la cantidad de datos e informaciones que se pueden aprovechar justifican que al esfuerzo en la obtención de datos se sume el que se realiza en la reflexión y la comprensión crítica de los datos obtenidos. Es la aplicación de la lógica dialéctica, la cual implica la consideración de los contrarios, alejándose de los planteamientos que dividen los métodos en cuantitativos y cualitativos que se propugna desde los planteamientos positivistas y constructivistas. El método dialéctico propuesto por la escuela de Frankfurt propugna el paso de la cantidad a la calidad, planteando la necesidad de una actitud analítico-sintética respecto al objeto de estudio basando su estrategia en un trabajo de interpretación. Interpretar, dirá Adorno (1973), "significa, ante todo, percibir la totalidad en los rasgos de la inmediata realidad social”.

Y para apoyar ese trabajo de interpretación, la investigación crítica también se apoya en la entrevista en profundidad a expertos o profesionales que pueden dar cuenta del entorno que conocen y en el que trabajan. En 30 ocasiones se utiliza esta técnica de investigación, que apoya además la necesidad de dotar de base empírica el esfuerzo investigador, una exigencia cada vez más presente no solo en tesis doctorales sino también en proyectos de investigación competitivos.

\section{Discusión y conclusiones}

La investigación en comunicación que se desarrolla en España implica el esfuerzo personal de profesores que a su labor académica suman el trabajo investigador, una labor que supone un esfuerzo intelectual de alto nivel y un trabajo material que requiere movilizar recursos a veces inexistentes, como en estos tiempos de crisis. Por ello, cada contribución merece el reconocimiento público y cada investigación encuentra en su singularidad el mérito de contribuir al gran edificio del saber. Al tiempo, el conjunto de las investigaciones resultantes puede arrojar otras características al balance de una década de investigación sobre comunicación en España, objetivo principal que se marca el proyecto Mapcom y este trabajo en particular.

Y lo primero que se puede decir del estudio realizado es que ha habido una línea de investigación que analiza los fenómenos comunicativos como industrias peculiares, como industrias culturales. El concepto de Adorno y Horkheimer sigue teniendo vigencia más de 70 años después, dado queel 7,5\% de la investigación sobre comunicación se realiza desde la perspectiva teórica que utiliza en el análisis de los 
fenómenos comunicativos los supuestos actualizados de la escuela de Frankfurt: la Economía Política de la Comunicación.

Esta perspectiva se presenta como una propuesta teórica válida y heurísticamente potente para investigar la forma y la organización del sistema comunicativo de las sociedades actuales, principal objeto de estudio de este tipo de investigación, aunque también se investigan otros fenómenos de manera destacada, como la incidencia de las nuevas tecnologías en los fenómenos comunicativos, los medios como industrias 0 el mercado de la comunicación. Estos objetos contrastan con los que priorizan otras perspectivas y aun el conjunto de la investigación en comunicación pues, como señalan González Samé, Romero y Aguaded (2017: 440) "gran parte del estudio científico de la comunicación de masas a escala mundial y en Latinoamérica ha girado alrededor de las audiencias y del impacto de los mensajes".

La investigación crítica se caracteriza, además, por centrar el esfuerzo investigador en el audiovisual mediático. Un cambio que responde a la importancia de lo audiovisual en los últimos años, considerándolo ya como un medio propio, dada la convergencia multimedia existente, aunque todavía tienen un papel protagónico los medios convencionales: la radio, el cine y, sobre todo,la televisión. Ello contrasta con los medios preferentes de otro tipo de acercamiento, pues “la investigación española sobre comunicación realizada en la etapa más reciente se ha centrado muy mayoritariamente en el estudio del periodismo y la información periodística, del que se ocupan casi la mitad de los artículos publicados entre 2008 y 2014 en las revistas científicas" (Martínez Nicolás y Saperas, 2016). En este acercamiento, la prensa solo ocupa un cuarto de la investigación analizada.

En cuanto a la metodología de investigación, los resultados del estudio permiten concluir que las investigaciones desde la perspectiva de la Economía Política de la Comunicación, sean comunicaciones, tesis doctorales, artículos de investigación o proyectos competitivos, se caracterizan por una utilización escasa de los métodos más usados en investigación social, tanto en la versión cuantitativa, por el escaso peso de la observación sistemática o de la encuesta; como de la cualitativa, anecdótico el uso de la observación participante e inexistente el uso del focus group. Solo se detecta cierta importancia de la entrevista en profundidad en combinación con métodos documentales, los más utilizados en esta perspectiva, en su versión más convencional, es decir, como herramienta documental y no tanto aplicando las técnicas de análisis de contenido o del discurso que tanto se utilizan en los estudios mediáticos. 
La sombra en este tipo de estudios es la persistencia todavía de cierta brecha de género entre los investigadores, una diferencia ya superada en el conjunto de la investigación en comunicación en nuestro país. Al mayor peso de los investigadores varones en el conjunto de la investigación desde la EPC se suma la constancia del techo de cristal. Ese techo se manifiesta no solo en la investigación, Repiso; Berlanga, Said-Hung y Castillo (2020:9)también han constatado la diferencia de género en las cátedras de comunicación; pero en los estudios desde esta perspectiva existen 30 puntos de diferencia. Esta pauta se puede explicar acudiendo a la diferencia que ha marcado tradicionalmente la separación entre los diferentes temas, pues la política y la economía, ámbitos de poder, se han caracterizado por su preeminencia masculina. Pero esta explicación, en el siglo XXI, no se sostiene, sobre todo cuando se reivindica un espíritu crítico que destaca y reclama un mayor protagonismo femenino. Y esa reivindicación no solo debe realizarse para los objetos que se investigan, también se debería reclamar en la práctica de su investigación, toda vez que, como señala Mosco (2006: 60) "la praxis social, o la unidad fundamental de pensar y hacer, también ocupa un lugar central en la economía política de la comunicación”.

\section{Bibliografía}

Adorno, Theodor y Popper, Karl (1973): La disputa del positivismo en la sociología alemana. Grijalbo.

Almirón, Nuria (2009, $1^{\text {a }}$ ed. 1944): “Economía Política y Comunicación: una aproximación epistemológica a los orígenes”. Revista Latina de Comunicación Social, 65, 563-571.

http://www.revistalatinacs.org/09/art/46_845_ULEPICC_01/38Almiron.html

Almiron, Núria; Reig, Ramón (2007); "The communications research in Spain: The political economy epistemological approach". American Communication Journal, 9, n.2.

Artero, Juan Pablo (2015): "Economía y empresa de comunicación: escuelas académicas y períodos de desarrollo" En Austral Comunicación, 4, n.1. 
Barranquero, Alejandro y Limón, Nieves (2017): “Objetos y métodos dominantes en comunicación para el desarrollo y el cambio social en las Tesis y Proyectos de Investigación en España (2007-2013)". Revista Latina de Comunicación Social, 72, 1-25. http://www.revistalatinacs.org/072paper/1151/01es.html

Bustamante, Enrique (2002): Comunicación y cultura en la era digital: industrias, mercados y diversidad en España. Gedisa.

Caffarel-Serra, Carmen; Domínguez, Milagros; Romano, Vicente (1989): "El estado de la investigación de Comunicación en España (1978-1987)”. Cinco. Cuadernos de investigación en comunicación, n. 3, 45-57.

Caffarel, Carmen; Ortega, Félix y Gaitán, Juan-Antonio (2017): "Investigación en comunicación en la universidad española en el periodo 2007-2014”. En El profesional de la Información, 26, $\mathrm{n}^{\circ} 2$.

https://recyt.fecyt.es/index.php/EPI/article/view/epi.2017.mar.08/34470

Carrasco, Andrés y Saperas, Enric (2013): “Industrias culturales" y "políticas de comunicación": bases conceptuales de la operacionalización institucional de las corrientes críticas de investigación social”. En Ramón Zallo y Andreu Casero-Ripollés (eds): Comunicación y regeneración democrática. Actas del IV Congreso Ulepicc.

Carrasco, Angel (2016): “La recepción de la Teoría Crítica en la investigación de la comunicación en España: contexto académico, tradiciones intelectuales y esfuerzos editoriales". V Congreso Internacional de la AEIC.

De Aguilera, Miguel (1998): “La investigación sobre Comunicación en España: una visión panorámica”. Comunicación y cultura, n. 4, 5-12.

https://dialnet.unirioja.es/servlet/articulo? codigo=2901273

Del Rincón, Delio, Arnal, Justo; Latorre, Antonio y Sans, Antoni (1995): Técnicas de investigación en ciencias sociales. Madrid: Dykinson.

Díaz-Nosty, Bernardo y De Frutos, Ruth (coords.) (2016): Rumbos de la investigación en España sobre Comunicación. Universidad de Málaga

Fernández-Quijada, David; Masip, Pere (2013): "Tres décadas de investigación española en Comunicación: hacia la mayoría de edad". Comunicar, n. 41, 15-24. https://doi.org/10.3916/C41-2013-01 
Fuchs, Christian (2009): Teoría crítica de la Información, la comunicación, los medios y la tecnología. En Glossarium Bitrum

http://glossarium.bitrum.unileon.es/

Galletero, Belén (2018): Del periódico impreso al diario digital: estudio de una transición en Castilla-La Mancha. Tesis doctoral, Universidad Castilla la Mancha.

González Morales, Alfredo (2003): “Paradigmas de investigación en ciencias sociales". Islas, 45, 125-135.

http://islas.uclv.edu.cu/index.php/islas/article/view/617

González Samé, Héctor; Romero, Luis M. y Aguaded, Ignacio (2017): “La investigación en comunicación en Latinoamérica: una aproximación histórica (1950-2016)”. Historia y comunicación social, 22, n. 2, 427-445.

Horkheimer, Max y Adorno, Theodor (1994, $1^{\text {a }}$ ed. 1944): Dialéctica de la Ilustración. Trotta.

Jeffries, Stuart (2018): Gran Hotel Abismo. Biografía coral de la Escuela de Frankfurt. Taurus.

Jones, Daniel E. (1998): “Investigación sobre Comunicación en España. Evolución y perspectivas". ZER, 3, n. 5, 13-51.

https://ojs.ehu.eus/index.php/Zer/article/view/17359

Lozano, Carlos; Gaitán, Juan Antonio; Caffarel, Carmen; Piñuel, Jose Luis (2020). "Una década de investigación universitaria sobre Comunicación en España, 20072018". El Profesional de la información, 29, n. 4.

https://doi.org/10.3145/epi.2020.jul.12

Martín Algarra, Manuel; Serrano-Puche, Javier y Rebolledo, Marta (2018): "La mujer en la investigación en comunicación en España: un análisis de la producción científica (2007-2013)". En: AdComunica. Revista Científica de Estrategias, Tendencias e Innovación en Comunicación, 15. 68-87.

http://dx.doi. org/10.6035/2174-0992.2018.15.5

Martínez Nicolás, Manuel (2009): "La investigación sobre comunicación en España. Evolución histórica y retos actuales”. En Revista Latina de Comunicación Social, 64. 
Martínez Nicolás, Manuel y Saperas, Enric (2016): “Objetos de estudio y orientación metodológica de la reciente investigación sobre comunicación en España (20082014)". En Revista Latina de Comunicación Social, 71.

Medina, Ileana (1998): “Los estudios sobre comunicación masiva en América Latina” En Revista Latina de Comunicación Social, 1.

Montero, Ma Dolores (1994): La información periodística y su influencia social. Ed. Labor/U.A.B.

Moragas, Miquel de (1988): "Los estudios sobre comunicación y nuevas tecnologías en España: indicaciones sobre sus antecedentes y estado actual”, en Cinco, 1, 11-19.

Mosco, Vicent (2006) "La Economía Política de la Comunicación: una actualización diez años después". Cuadernos de la Información y la Comunicación, 11, 57-79.

Neuman, W. Laurence (1994): Social Research Methods. Qualitative and Quantitative Approaches. Allyn and Bacon.

Popper, Karl (2006, $1^{\text {a }}$ ed.1961): Miseria del historicismo. Alianza Editorial.

Repiso, Rafael; Berlanga, Inmaculada; Said-Hung, Elías; Castillo, Antonio (2020): “Titularidad y cátedras en Comunicación en España (2000-2019). Distribución, ritmos de promoción, transferencia entre universidades y endogamia”. Profesional de la información, 29, n. 4. https://doi.org/10.3145/epi.2020.jul.22

Rodrigo Alsina; Miquel (2014): “La sociosemiótica como método de investigación en periodismo". en Brazilian Journalism Research, 11, n. 2.

Saperas, Enric (1998): Manual básico de teoría de la comunicación. CIMS.

--- (2016): “Cuatro décadas de investigación comunicativa en España. Los procesos de institucionalización y de profesionalización de la investigación (19712015)". Disertaciones, 9, n. 2, 27-45.

https://doi.org/10.12804/disertaciones.09.02.2016.02 
Sierra, Francisco (2009): "Economía política de la comunicación y teoría crítica. Apuntes y tendencias" Revista Científica de Información y Comunicación 6, 149-171

Van Dijk, Teun (2002): "El análisis crítico del discurso y el pensamiento social." Athenea digital, 1, 18-24.

https://atheneadigital.net/article/view/n1-van 